\title{
Semiconductor quantum dots: Theory and phenomenology
}

\author{
VIJAY A SINGH*, V RANJAN and MANISH KAPOOR ${ }^{\dagger}$ \\ Physics Department, Indian Institute of Technology, Kanpur 208 016, India \\ †Physics Department, D.A.V. College, Kanpur 208 001, India
}

\begin{abstract}
Research in semiconductor quantum dots (q-dots) has burgeoned in the past decade. The size $(R)$ of these q-dots ranges from 1 to $100 \mathrm{~nm}$. Based on the theoretical calculations, we propose energy and length scales which help in clarifying the physics of this mesoscopic system. Some of these length scales are: the Bohr exciton radius $\left(a_{\mathrm{B}}^{*}\right)$, the carrier de Broglie and diffusion length $\left(\lambda_{\mathrm{D}}\right.$ and $\left.l_{\mathrm{D}}\right)$, the polaron radius $\left(a_{\mathrm{p}}\right)$, and the reduction factor modulating the optical matrix element $\left(M_{x}\right), R<a_{\mathrm{B}}$ is an individual particle confinement regime, whereas the larger ones are exciton confinement regime wherein Coulomb interaction play an important role. Similarly a size-dependent dielectric constant $\varepsilon(R)$ should be used for $R<a_{\mathrm{p}}<a_{\mathrm{B}}$. An examination of $M_{x}$ reveals that an indirect gap material q-dot behaves as a direct gap material in the limit of very small dot size. We have carried out effective mass theory (EMT) calculations to estimate the charge density on the surface of the quantum dot. We present tight binding (TB) calculation to show that the energy upshift scales as $1 / R^{x}$, where $x$ is less than 2 and the exponent depends on the orientation of the crystallite.
\end{abstract}

Keywords. Semiconductor; quantum dots; confinement; tight-binding; phenomenology.

\section{Introduction}

Work on semiconductor nanostructures began about two decades ago (Brus 1986; Wang and Herron 1991; John and Singh 1995). It received a fillip in 1990 when Canham (1990) discovered that nanocrystalline porous silicon yields efficient photoluminescence in the visible range. Alongside there was also an inexorable technological drive towards using increasingly smaller devices. The technology is currently in the submicron stage. The optimistic view that the faster, nanoelectronic devices could become available in the next decade is not without a basis.

In the present work we have attempted to describe theoretical and phenomenological work being carried out in this field. In $\S 2$ we have described phenomenological scaling laws in this field. This section can as well be used as a primer (introductory) by those wishing to acquaint themselves with this exciting and emerging field. In $\S 3$ we have described methods which yield electronic spectra of the semiconductor nanostructures. The two main approaches described are: EMT and TB. $\S 4$ encapsulates our conclusions.

\section{Phenomenological scaling laws}

In this section we propose to discuss some scaling laws that set mesoscopic systems apart from both the micro-

*Author for correspondence scopic as well as the bulk. In a sense these scaling laws are a signature of a whole class of these systems. These may provide an initial hint to the emerging form of physical laws valid for this intermediate class of materials. We shall state and motivate scaling laws in terms of the following parameters: (i) size (diameter $d$ ) with $d \propto[1-10 \mathrm{~nm}]$; (ii) dimensionality, $D,(0$ for a dot); (iii) energy, $E$; and (iv) temperature, $T$. We have divided the scales into (i) quantum scales, and (ii) classical scales.

\subsection{Quantum scales}

Size: The foremost question is: 'What yardstick should be followed to define a semiconductor mesoscopic system?' We proffer the following candidates for length scales in these materials:

(I) Bulk Bohr exciton radius: The Bohr radius $\left(a_{\mathrm{B}}^{*}\right)$ of the exciton in bulk semiconductors is given by

$$
a_{\mathrm{B}}^{*}=\frac{\hbar^{2} \varepsilon}{e^{2}}\left(\frac{1}{m_{\mathrm{e}}^{*}}+\frac{1}{m_{\mathrm{h}}^{*}}\right) \text {, }
$$

where $m_{\mathrm{e}}^{*}\left(m_{\mathrm{h}}^{*}\right)$ are the electron (hole) effective masses and $\varepsilon$ is the relative dielectric constant of the material. This sets a natural size scale. If the diameter $d$ of the semiconductor nanostructure is less than $a_{\mathrm{B}}^{*}$ then the material is said to be in the strong confinement regime, and when the diameter is larger than $a_{\mathrm{B}}^{*}$ it is said to be in the weak confinement regime. 
(II) Bulk absorption length $\left(l_{\mathrm{a}}\right)$ : Optical absorption is one of the important experimental methods for the characterization of a material. Absorption length $l_{\mathrm{a}}$ can naturally define another length scale. From Beer's law we obtain

$$
I=I_{0} \exp \left(-d / l_{\mathrm{a}}\right)
$$

If the size is much larger than the absorption length $\left(d>l_{\mathrm{a}}\right)$, light is absorbed and information can be obtained on the diffusion length of the charge carriers generated by the photons. However, if the size is smaller than the absorption length, no absorption spectrum is possible and instead information on the relaxation time of the charge carriers can be obtained.

(III) Diffusion length: The bulk diffusion length $\left(l_{\mathrm{D}}\right)$ is defined in terms of the diffusion constant $D$ and relaxation time $\tau$ as:

$$
l_{\mathrm{D}}=\sqrt{D \tau},
$$

where $D$ is given by Einstein relation,

$$
D=\frac{\mu k T}{e} .
$$

Here $\mu$ is the mobility of the carrier. An electron diffuses a certain distance inside the material before radiatively recombining with a hole. The distance traversed is called the diffusion length. A large diffusion length means that the electron encounters the surface several times before recombination. This implies a high probability for nonradiative capture of electrons by uncapped surface bonds. Efficient luminescence being the hallmark of semiconductor nanostructures, the bulk diffusion length plays an important role in these systems.

Energy scale: In contrast to the bulk ( $\sim 10^{23}$ atoms), nanostructures $\left(\sim 10^{3}\right.$ atoms) have discrete eigen spectra. Hence they are called artificial atoms or discrete materials.

(I) HOMO-LUMO gap: In such a discrete eigen spectrum the analogue of the valence band maximum (VBM) is known as highest occupied molecular orbital (HOMO) and the analogue of the conduction band minimum (CBM) is known as the lowest unoccupied molecular orbital (LUMO). The HOMO-LUMO gap is the band gap. The gap $E_{\mathrm{g}}(d)$ scales as (figure 1)

$$
E_{\mathrm{g}}(d) \propto \frac{1}{d^{\beta}} .
$$

Different theories yield different values of $\beta$, but all predict it to be in the range $\beta \in[1,2]$.

A simple model of a particle in a spherical box of diameter $d$ (figure 1) will yield $\beta$ to be equal to 2 . In the ground state of particle inside such a box: $\lambda / 2=d$.
Hence the deBroglie relation defines momentum as: $p=h / \lambda=h / 2 d$. The kinetic energy of confinement for this particle is given as $p^{2} / 2 m=h^{2} /\left(8 m d^{2}\right)$. Consequently this simple example demonstrates that the exponent $\beta=2$.

(II) Mean energy level spacing ( $\Delta)$ : Since the eigen spectrum is discrete, the spacing between two levels $(\Delta)$ may be less or more than the energy $k T, T$ being the room temperature. While $\Delta \ll k T$ corresponds to the bulk, $\Delta \simeq k T$ corresponds to mesoscopic systems. Some interesting physics is possible on the basis of this observation. An example of very low energy transitions in materials are phonon-assisted transitions. But because of the discrete spectrum of such systems, a phononassisted transition may be disallowed if the phonon energy does not match the energy difference of the two states.

In figure 1 we have shown an idealized density of states (DOS) vs energy $(E)$ diagram for a quantum dot of diameter $d$. The mean energy spacing $\Delta$ is shown and the band gap is shown as the HOMO-LUMO gap. The figure demonstrates why nanostructures are called digital or discrete materials. Since, the DOS is similar to the one for atoms, hence these materials are also called artificial atoms.

Luminescence: Oscillator strength and relaxation time are important factors in determining the efficiency of luminescence from such materials.

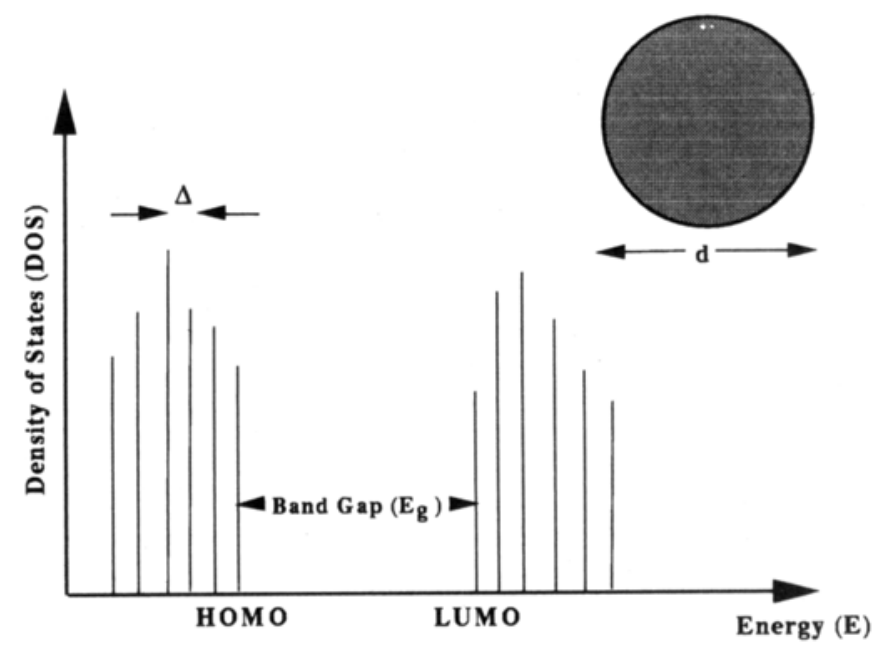

Figure 1. An idealized density of states (DOS) for a semiconductor dot. The spectrum is discrete with mean inter-level spacing $\approx \Delta$. The analogue of the valence band maximum (VBM) is the highest occupied molecular orbital (HOMO) and that of conduction band minimum (CBM) is the lowest unoccupied molecular orbital (LUMO). States below (above) HOMO (LUMO) are filled (empty) at $T=0 \mathrm{~K}$. On the top of the figure we display an idealized semiconductor dot of spherical shape and diameter $d$. 
(I) Oscillator strength: Larger coupling strength will mean a proportional number of electrons recombining with holes, hence higher intensity will arise from transition between two states.

Optical matrix element $\left(\left|M_{x}\right|\right)$ is found to scale with size (Hybertsen 1992) as:

$$
\left|M_{x}\right| \propto \frac{1}{d^{3}}
$$

Hence intensity of the luminescence will be higher for smaller sized crystallites.

(II) Radiative time $\left(\tau_{\mathrm{r}}\right)$ : The radiative time scales as:

$$
\tau_{\mathrm{r}} \propto\left|M_{x}\right|^{-2}
$$

This implies a very strong polynomial dependence on size. Thus, the radiative rate $\left(1 / \tau_{\mathrm{r}}\right)$ and consequently the radiative efficiency are strongly enhanced in nanocrystallites.

Thus, (5) and (6), therefore imply an efficient blueshifted luminescence from nanocrystallites.

\subsection{Classical scaling laws}

Surprisingly some classical scaling laws also assume significance for nanostructures. Some of these are:

(I) Surface to volume ratio $(S / N)$ : Since the relation between surface area $\left(S \sim d^{R}\right)$ and volume $\left(V \sim d^{\beta}\right)$ is given by,

$$
\frac{S}{V} \propto \frac{1}{d}
$$

therefore, this implies larger $S / V$ for smaller clusters. As a consequence the smaller the cluster the larger the proportion of atoms on the surface. For example, about $15-30 \%$ atoms are on the surface in a $50 \AA \mathrm{CdS}$ cluster (Wang and Herron 1991), i.e. around 1000 atoms of a total of $3000-4000$ are on the surface. This may mean a proportionately large number of dangling bonds on the surface and consequently a large number of dangling bonds may mean higher probability for non-radiative recombination. Hence surface effects need careful examination in a nanostructure.

(II) Power requirement $(P)$ : Power requirement scales like $P \sim \not{d}$. Hence devices made of nanostructures would be expected to operate with a miniscule amount of power.

(III) Wear life (W): Wear life is proportional to the thickness $(d)$ and inversely proportional to the erosion rate. Erosion rate scales with surface area $\left(\sim d^{2}\right)$. Hence the wear life will scale as $1 / d$.

\section{Electronic structure}

\subsection{Effective mass theory}

A semiconductor nanostructure is generally embedded in a large band gap matrix (glass, polymer, zeolites, etc). The dielectric constitutes a large but finite barrier $V_{0}$ to the electron-hole pair in the nanostructures. The bound state of the electron-hole pair (Wannier exciton) may be described by the hydrogenic Hamiltonian as:

$$
\begin{aligned}
& H=-\frac{\hbar^{2}}{2 m_{\mathrm{h}}^{*}} \nabla_{\mathrm{h}}^{2}-\frac{\hbar^{2}}{2 m_{\mathrm{e}}^{*}} \nabla_{\mathrm{e}}^{2}-\frac{e^{2}}{\varepsilon\left|\overrightarrow{r_{\mathrm{e}}}-\overrightarrow{r_{\mathrm{h}}}\right|}+V, \\
& V= \begin{cases}0 & r<R(=d / 2) \\
V_{0} & r>R\end{cases}
\end{aligned}
$$

Here $m_{c}^{*}\left(m_{h}^{*}\right)$ is the electron (hole) effective mass and $\overrightarrow{r_{\mathrm{e}}}\left(\overrightarrow{r_{\mathrm{h}}}\right)$ is the electron (hole) coordinate. The first two terms in (8) in the Hamiltonian represent the kinetic energy of the electron and the hole. The third term is the Coulomb potential energy where $\varepsilon$ is the relative dielectric constant. The last term represents the effect of the surrounding dielectric.

The above Hamiltonian is commonly solved by the variational method, using a trial wavefunction:

$$
\psi\left(\overrightarrow{r_{\mathrm{e}}}, \overrightarrow{\mathrm{r}_{\mathrm{h}}}\right)=N \phi\left(\overrightarrow{r_{\mathrm{e}}}\right) \phi\left(\overrightarrow{r_{\mathrm{h}}}\right) \exp \left(-\overrightarrow{r_{\mathrm{eh}}} / \alpha\right)
$$

where $N$ is the normalization constant and $\alpha$ the variational parameter. The exponential term represents electron-hole correlation. Within this simplified model, the band gap $E_{\mathrm{g}}(d)$ is typically obtained as

$$
E_{\mathrm{g}}(d)=E_{\mathrm{g}}^{\mathrm{buik}}+\frac{c_{1}}{d^{2}}-\frac{c_{2}}{d}-c_{\gamma} E_{\mathrm{Ry}}^{*} .
$$

Here the second term on the right hand side is the kinetic energy which is due to confinement of the electron and the hole. It implies a blue-shift with respect to the bulk bandgap. The third term is due to the Coulomb attraction between electron and hole and the last term is the bulk exciton binding energy written in terms of Rydberg energy $E_{\mathrm{Ry}}$. The other symbols $c_{1}, c_{2}$ and $c_{y}$ represent constants. Such an analysis was first carried out by Brus (1983) and Efros and Efros (1982). Based on the above analysis Efros and Efros (1982) proposed the following widely accepted classification of semiconductor nanostructures:

(I) Strong confinement $\left(R<a_{\mathrm{B}}^{*}\right)$ : When the radius of the crystallite is smaller than the bulk Bohr exciton radius, the confinement energy $\left(1 / d^{2}\right)$ dominates over the Coulomb energy $(-1 / d)$. Hence the Coulomb correction to the bulk band gap may be ignored and the electron 
and the hole are said to be weakly correlated (individual particle confinement regime).

(II) Weak confinement $\left(R>a_{\mathrm{B}}^{*}\right)$ : When the radius of the crystallite is larger than the bulk Bohr exciton radius, the Coulomb energy $(-1 / d)$ dominates over the confinement energy $\left(1 / d^{2}\right)$. Hence the correction to the bulk band gap due to confinement energy may be ignored. The electron and the hole are said to be strongly correlated (exciton confinement regime).

In the past two decades a great deal of work has been carried out in refining the above calculation and relating them to realistic systems. An obvious improvement is on the trial wavefunction in (9). A multiparameter wavefunction (Nair et al 1987) and an expanded basis set (Kayanuma 1988) have been used. An improved Hamiltonian taking into account nonparabolicity of the conduction band (Nomura and Kobayashi 1991) and the degenerate nature of the valence band (Takagahara and Takeda 1992; Laheld and Einevoll 1997) has been considered. Non-spherical crystallites with cubical (Hybertsen 1992) and cylindrical (Voos et al 1992) shapes have been considered. Silicon crystallites where the band gap is indirect require a separate treatment (Hybertsen 1992; Voos et al 1992; Khurgin et al 1996). The size dependence of the dielectric constant $(\varepsilon(d))$ has been discussed. Multiexcitonic effects have also been discussed by Nair and Takagahara (1997). There is still much scope for improvement and extending this scheme. One such example is the recent work by Bhattacharjee and Guillaume (1997) where they have considered an excitonic Hamiltonian due to the formation of magnetic polaron in diluted magnetic semiconductors.

It is also possible to study multi-electron effects in these systems. The energy required to add an electron to the dot can be computed and this serves to define the capacitance of the dot (Ashoori 1996).

We conclude this section by mentioning an aspect that has not been discussed in literature. If the electron effective mass in the crystallite is very different from the one in the dielectric, then we have observed that the charge density is very large on the surface (figure 2). This implies that the electron will largely reside on the surface, get trapped by dangling bonds or chemical complexes and be prevented from radiative recombination.

\subsection{Tight-binding}

The tight-binding (TB) scheme is one of the most popular methods for electronic structure calculation of semiconductor clusters. This scheme employs a linear combination of atomic orbitals (LCAO) to solve the Schröedinger equation for a cluster of atoms. The size of the cluster can be varied by adding atoms shell by shell. Different surface geometries can be selectively studied.
We have performed a TB calculation on silicon clusters of sizes up to $40 \AA$. The scheme, we have followed, has the following features: (i) 4-orbitals per site; (ii) nonorthogonal orbitals; and (iii) many neighbour interactions.

Since the crystallites are of extremely small size, they have a large surface to volume ratio. Hence we expect that the surface orientation plays an important role in determining the electronic structure and the consequent opto-electronic properties. The surface orientation is varied by suitably selecting the central site. We have performed these calculations for four different surface geometries: (i) bond-centred (BC); (ii) hexagonal-centred (HC); (iii) tetrahedral-centred (TC); (iv) substitutionalcentred (SC).

As mentioned in $\S 2.2$ in the discussion following (7), there are large number of dangling bonds $\left(N_{\mathrm{d}}\right)$ on the surface of a small cluster $\left(N_{\mathrm{d}} \propto d^{2}\right)$. In our calculation this fact is not only revealed but for hydrogen passivated clusters the proportion of monohydrides is found to be much more than the dihydrides and trihydrides.

We have performed TB calculations for passivated as well as unpassivated clusters. The dangling bonds are passivated with hydrogen. Since the dangling bonds form levels deep in the gap, the band gap in our calculation is observed to be less than the one for hydrogen-passivated clusters.

In figure $3 \mathrm{a}$ we have plotted the band gap variation of the bond-centred cluster (BC) with the size. We find that for the passivated clusters

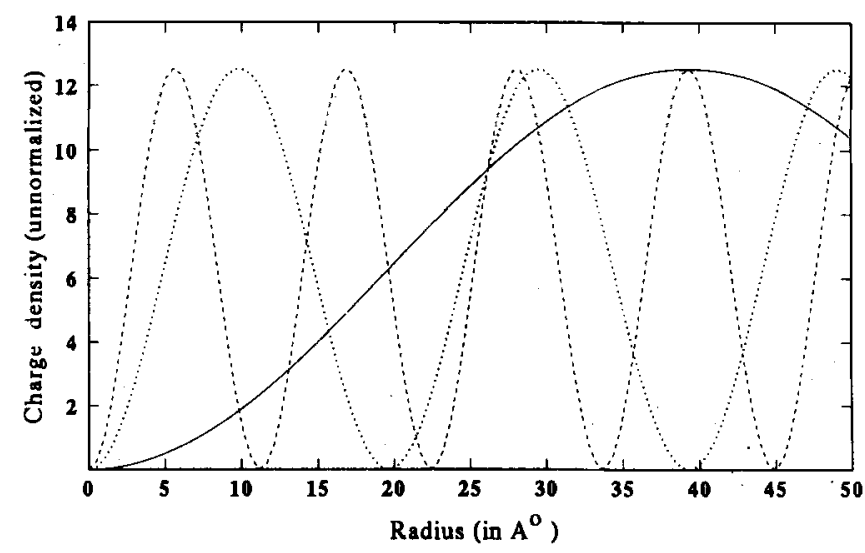

Figure 2. Charge density of an electron in a spherical quantum dot of radius $R=50 \AA$ as a function of distance from the center $(r)$ is plotted. A quantum well of size $R$ and depth $V_{0}=1.37 \mathrm{eV}$ represents the dot. The mass of the electron inside the well is 0.3 times the one outside the well. The charge density is defined as $4 \pi r^{2} \psi^{*} \psi$. In this plot we have used unnormalized wavefunctions $\psi$. Solid line (-) is for states with quantum numbers $n=1, l=0$. Dots (...) are for $n=3, l=0$ and the dashed line $\cdot(--) n$ is for $n=5, l=0$. It is observed that the charge density for all the above mentioned states has a large value at the boundary $(R=50)$. 

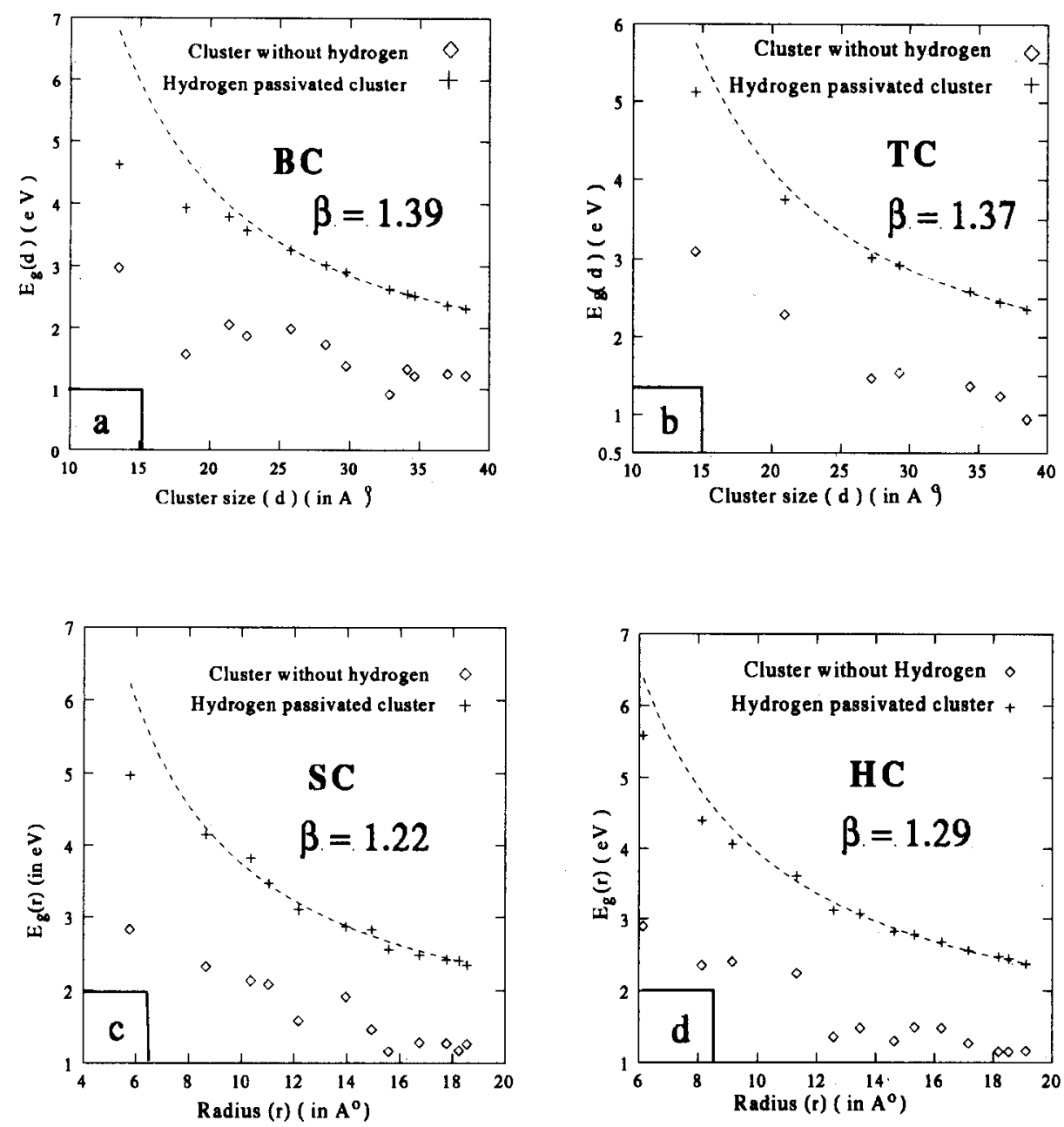

Figure 3. Energy dependence of a the bond-centred (BC), b the tetrahedral (TC), c substitutional (SC) and $\mathbf{d}$ the hexagonal (HC) cluster on cluster size $(r=d / 2)$. The details are described in $\$ 3.2$.

$$
E_{\mathrm{g}}(d)=E_{\mathrm{g}}^{\mathrm{bulk}}+\frac{c}{d^{\beta}}
$$

with the exponent $\beta=1.51$ and $c=288.37$ in appropriate units. In the above equation $E_{\mathrm{g}}^{\text {bulk }}$ is the band gap of bulk silicon $(1.17 \mathrm{eV})$. It is clear from the figure that the band gap asymptotically approaches the bulk value as the cluster size is increased. We note that the passivated clusters have a larger band gap. Because the dangling bonds introduce extra states in the gap, we obtain a lower gap for unpassivated clusters. Figure $3 b$ is a similar plot for tetrahedral clusters (TC). But the exponents in (11) for the passivated TC is $\beta=1.37$ and $c=178.76$ in appropriate units. The exponent $\beta=1.22$ for the passivated substitutional-centred (SC) and 1.29 for hexagonal-centred (HC) clusters as shown in figures $3 c$ and $d$, respectively.

We find that the enhancement of the band gap is weaker than that predicted by EMT. For the latter $\beta=2$. The orientational dependence of the gap is also discernible. Tight binding calculations of Delerue et al (1993) for the SC cluster yield $\beta=1.39$. In phenomenological studies on luminescence (John and Singh 1995; Ranjan and Singh 1998), a weaker dependence of gap enhancement must be borne in mind. Our study also indicates that the visible luminescence is from passivated clusters. The unpassivated clusters yield reduced gaps which are in the infra-red for cluster sizes $d \geq 3 \mathrm{~nm}$.

Many other workers have performed TB calculations on semiconductor nanostructures. In their work many variants of the TB scheme have been used. They are all semi-empirical approaches. These different schemes differ in the use of the following: (i) orthogonal or nonorthogonal orbitals; (ii) the number of nearest neighbour interaction; and (iii) the number of orbitals per site.

The Vogl model is an empirical nearest neighbour, orthogonal, five-orbitals $\left(s p^{3} s^{*}\right)$ TB approach. This scheme involves a reduced computational requirement and hence is popularly used. But it produces (incorrectly) a flat conduction band (Delerue et al 1996). 
As we have mentioned earlier in this section, we have used many neighbour, nonorthogonal, four orbitals TB scheme. Some other workers have also used similar schemes (Menon and Subbaswamy 1997). However within these schemes, too, there is a variation of methods in as far as the choice of other parameters is concerned.

These schemes have been adopted for various systems. Some workers have carried out studies on group II-VI materials, namely $\mathrm{CdS}, \mathrm{CdSe}, \mathrm{ZnO}$ (Lippens and Lannoo 1989; Wang and Herron 1991). Others have carried out studies on Si-nanostructures. Allan et al (1997) have studied the amorphous semiconductors $(a-\mathrm{Si})$ and hydrogen passivated $a$-Si $(a-S i: H)$ (Allan et al 1997a) and temperature studies of these materials (Allan et al 1997b). A simplified method called effective bond orbital method (EBOM) has been used by some to study these materials (Ramaniah and Nair 1993; Laheld and Einevoll 1997). Filonov et al (1997) have used self-consistent extended Huckel theory (EHT) to study novel structures, pseudofilm, pseudowire and pseudodot, of silicon (Filonov et al 1997).

\section{Conclusion}

In the preceding sections we have discussed the two main approaches to calculate electronic structure of semiconductor nanostructures. We note here that the TB method calculates only the confinement energy correction in the nanostructures, whereas EMT calculates both the confinement energy and Coulomb energy corrections. Nevertheless, even in the calculation of confinement energy both methods differ in a fundamental manner. EMT predicts the confinement energy to scale as $\sim 1 / d^{2}$, whereas the TB predicts it to scale as $\sim 1 / d^{\beta}$, where $\beta \in[1,2]$. The EMT result of (10) can be rewritten as

$$
E_{\mathrm{g}}(d)=E_{\mathrm{g}}^{\mathrm{bulk}}+\frac{c^{\prime}}{d^{\alpha}}
$$

the value of $\alpha$ is always found to be between 1 and 2 .

As mentioned in $\$ 3.2$, there are a variety of TB schemes used for electronic structure calculation. It is reasonable to demand that the TB calculations satisfy the following elementary requirements (Delerue et al 1996):

(I) The transferability of the parameters from the known bulk properties to the unknown crystallite case. These bulk properties are usually the bulk band gap structure and its derivatives (hole and electron masses).

(II) The boundary conditions are proper. In other words the hydrogen terminators should mimic the large band gap of the material coating the crystallite.

Most TB calculations overlook these requirements.
For more than a decade a large number of experiments have been done to evaluate the band gap of semiconductor nanostructures and comparisons have been made with the theoretical results expressed in (10), (11) and (12). The experiments are photoluminescence (PL), photoluminescence excitation (PLE) spectra and absorption spectra. These are optical experiments but some nonoptical measurements like photoemission spectra have also been carried out. While there is no unanimity in the interpretation of experimental data, the blue-shift has been universally reported. The TB is almost always found to be in better agreement than the EMT. The latter is found to overestimate the band gap.

Semiconductor nanostructures must be viewed as mesoscopic disordered structures. With a few exceptions the electronic structure calculation described in $\$ 3$ do not take disorder into account. The work of Sawada et al (1994) on porous silicon, Filonov et al (1997) on novel structures in silicon and our own work in $\$ 3.2$ where different surface geometries are explored, represent attempts to address the issue of disorder. An interesting example is the recent work of Ranjan et al (1998). This work addresses the issue of luminescence for size distributed crystallites/dots. The dependence of the oscillator strength and the gap on size is obtained from the microscopic electronic structure calculations. This dependence is then used as a phenomenological input in the calculation of the PL spectra. It is demonstrated that the PL peak may be blue- or red-shifted depending on the competition and form of the scaling laws.

The conceptual development in the field of mesoscopic structures has been driven by a dialectical process in which tools from the microscopic and the macroscopic have been extensively and at times indiscriminately employed. The electronic structure calculations provide critical support to some of the phenomenological scales that we have described in $\S 2$. We believe that a fuller understanding of these materials will emerge by synthesizing the results of electronic structure calculations with the phenomenological laws.

\section{Acknowledgements}

The work was supported in part by the Department of Atomic Energy through the Board of Research in Nuclear Sciences (No. 37/11/97-R\&D II). Discussions with $\mathrm{Dr}$ Swapan Ghosh, BARC, Mumbai are gratefully acknowledged.

\section{References}

Allan G, Delerue C and Lannoo M 1997a Phys. Rev. Lett. 78 3161

Allan G, Delerue C and Lannoo M 1997b Appl. Phys. Lett. 71 1189

Ashoori R C 1996 Nature 379413 
Bhattacharjee A and Guillaume C 1997 Phys. Rev. B55 10613 Brus L E 1983 J. Chem. Phys. 795566

Brus L E 1986 J. Phys. Chem. 902555

Canham L T 1990 Appl. Phys. Lett. 571046

Delerue C, Allan G and Lannoo M 1993 Phys. Rev. B48 11024

Delerue C, Lannoo M and Allan G 1996 Phys. Rev. Lett. 76 3038

Efros Al L and Efros A L 1982 Sov. Phys. Semiconduct. 16 772

Filonov A et al 1997 Appl. Phys. Lett. 70744

Hybertsen M S 1992 Mater. Res. Soc. Symp. Proc. 256179

John G C and Singh V A 1995 Phys. Rep. 26393

Kayanuma Y 1988 Phys. Rev. B38 9797

Khurgin J B, Forsythe E W, Tompa G S and Khan B A 1996 Appl. Phys. Lett. 691241

Laheld U and Einevoll G 1997 Phys. Rev. B55 12311
Lippens P E and Lannoo M 1989 Phys. Rev. B39 10935 Menon M and Subbaswamy K 1997 Phys. Rev. B55 9231 Nair S V and Takagahara T 1997 Phys. Rev. B55 5153

Nair S V, Sinha S and Rustagi K C 1987 Phys. Rev. B35 4098 Nomura S and Kobayashi T 1991 Solid State Commun. 78677 Ramaniah L and Nair S 1993 Phys. Rev. B47 7132

Ranjan V and Singh V A 1998 in Proc. of the ninth int. workshop on the physics of semiconductor devices (eds) $\mathrm{V}$ Kumar and S K Agrawal (London: Narosa) pp 98-101

Ranjan V, Singh Vijay A and John George C 1998 Phys. Rev. B58 1158

Sawada S, Hamada N and Ookubo N 1994 Phys. Rev. B49 5236

Takagahara T and Takeda K 1992 Phys. Rev. B46 15578

Voos M et al 1992 Appl. Phys. Lett. 611213

Wang Y and Herron N 1991 J. Phys. Chem. 95525 